\title{
CONTRIBUIÇÃO DE FUNGOS MICORRÍZICOS ARBUSCULARES NATIVOS NO ESTABELECIMENTO DE ARISTIDA SETIFOLIA KUNTH EM ÁREAS DEGRADADAS DO CERRADO ${ }^{1}$
}

\author{
CARLOS ROMERO MARTINS ${ }^{2}$, JEANNE CHRISTINE CLAESSEN DE MIRANDA ${ }^{3}$ e LEO NOBRE DE MIRANDA ${ }^{4}$
}

\begin{abstract}
RESUMO - Para estudar a dinâmica de fungos micorrízicos arbusculares (MA) nativos, em solo de cerrado natural e de três áreas degradadas, foram efetuadas avaliações quantitativa e qualitativa desses fungos, nos períodos seco e chuvoso. Nessa avaliação incluíram-se parcelas experimentais, em uma das áreas degradadas, com calcário, turfa natural e torta de mamona. Em casa de vegetação conduziram-se dois experimentos para avaliar a contribuição desses fungos no estabelecimento da gramínea pioneira Aristida setifolia Kunth. No primeiro, utilizaram-se substratos das quatro áreas, esterilizados a vapor e ao natural; no segundo, foram utilizados substratos da área experimental esterilizados, com e sem aplicação de mistura de três fungos MA nativos. No campo, a densidade aparente nas áreas degradadas foi superior à do cerrado natural, e todas apresentaram alta acidez e baixa fertilidade. Os fungos MA ocorreram em todas as áreas; houve maior número de esporos e espécies no período chuvoso. Em casa de vegetação, os fungos MA nativos promoveram aumentos significativos na matéria seca da gramínea. Não houve efeitos significativos do calcário e dos insumos orgânicos no crescimento das plantas sem inoculação dos fungos MA. Entretanto, com a inoculação, ocorreu acréscimo significativo no crescimento da gramínea e diferenciação e maximização nos efeitos desses insumos.
\end{abstract}

Termos para indexação: micorriza, solo degradado, gramínea nativa, cascalheira, recuperação do solo.

\section{CONTRIBUTION OF NATIVE ARBUSCULAR MYCORRHIZAL FUNGI IN THE STABLISHMENT OF ARISTIDA SETIFOLIA KUNTH IN DEGRADED AREAS IN THE CERRADO}

\begin{abstract}
To study the dynamics of native AM fungi, in natural cerrado soil and three degraded areas, quantitative and qualitative avaliations were done, in the dry and rainy seasons. Those avaliations included field experiment plots from a degraded area with lime, natural peat and castor bean cake. Two greenhouse experiments studied the contribution of AM fungi to the stablishment of a spontaneous grass Aristida setifolia Kunth. The first used natural and steam sterilized substrates from the four areas; the second, field plots substrates steam sterilized with and without inoculation of three native AM fungi. In the field, soil density was higher in degraded areas and both areas presented high soil acidity. The AM fungi were present in all areas with higher number of spores and species in the rainy season. In the greenhouse experiments, native AM fungi promoted a significant increase in plant shoot dry matter. In the noninoculated field plots substrates, there was no significant effect of lime and organic compounds on plant growth. However, with the inoculation, there was a significant increase in grass shoot dry matter, with a significant improvement and differentiation of the field treatments effects.
\end{abstract}

Index terms: mycorrhiza, degraded soil, native grass, gravel bed, soil recovering.

${ }^{1}$ Aceito para publicação em 28 de setembro de 1998.

Extraído da Tese de Mestrado do primeiro autor, apresentada à Universidade de Brasília.

${ }^{2}$ Eng. Agr., M.Sc., IBAMA/SEDE-DIRCOF/DEREL/DIAP, SAIN, Av. L4 Norte, CEP 70800-200 Brasília, DF.

${ }^{3}$ Bióloga, Ph.D., Embrapa-Centro de Pesquisa Agropecuária dos Cerrados (CPAC), Caixa Postal 08223, CEP 73301-970 Planaltina, DF. E-mail: jeanne@cpac.embrapa.br

${ }^{4}$ Eng. Agr., Ph.D., Embrapa-CPAC. E-mail: leo@cpac.embrapa.br

\section{INTRODUÇÃO}

A perturbação e a degradação dos solos tropicais tem-se intensificado nas últimas décadas, principalmente pela mineração, construção civil e agricultura e pecuária intensivas quando realizadas de forma inadequada (Nepstad et al., 1991; Vilela et al., 1991; Instituto Brasileiro de Mineração, 1992). Essas práticas têm deixado extensas áreas desprovidas de 
cobertura vegetal e expostas às intempéries climáticas (Chiossi, 1982; Leite et al., 1992), sendo necessário recuperá-las para racionalizar o uso da terra $\mathrm{e}$ melhorar a qualidade ambiental. A reimplantação da vegetação nativa ou a adaptação de espécies exóticas possibilita a produção de matéria orgânica, bem como a recuperação da comunidade microbiana do solo, que tem papel fundamental nas transformações que equilibram e sustentam os ecossistemas naturais (Ruivo, 1993) e cultivados (Maschio et al., 1992).

A associação entre o desenvolvimento vegetal e a atividade microbiana pode ser um fator importante na recuperação dos solos degradados, pois mesmo quando profundamente alterados, eles podem manter uma comunidade microbiana ativa. Os fungos micorrízicos arbusculares, por exemplo, têm sido naturalmente encontrados nesses solos alterados (Maschio et al., 1992), e segundo Allen \& Allen (1984), os fungos MA nativos podem interferir na composição, na competição e na sucessão das comunidades vegetais. A maioria das plantas que ocorrem espontaneamente em áreas mineradas apresentam simbiose com fungos MA e, conseqüentemente, são beneficiadas no seu crescimento e sobrevivência (Allen \& Allen, 1980). Veenendaal (1991), que estudou estratégias de adaptação de plantas, nas savanas semi-áridas degradadas de Botswana (África), constatou que a presença da simbiose com os fungos MA nativos foi fundamental para o estabelecimento de espécies de gramíneas, como as do gênero Aristida.

O estabelecimento e o cultivo de plantas nos solos degradados de cerrado requerem também a utilização de insumos químicos e orgânicos (Leite et al., 1994). Entretanto, a magnitude dos benefícios conferidos pela micorriza nessas condições pode estar relacionada ao manejo desses insumos. Tem sido observado que a contribuição dos fungos MA no crescimento de plantas em solos de baixa fertilidade como os de cerrado depende da utilização de calagem e adubação adequadas (Miranda \& Miranda, 1997).

Este trabalho foi desenvolvido com o objetivo de estudar a dinâmica dos fungos micorrízicos arbusculares nativos e a sua contribuição no estabelecimento e no crescimento de plantas em áreas degradadas de cerrado, com e sem a utilização de calcário e insumos orgânicos.

\section{MATERIAL E MÉTODOS}

Inicialmente, foi efetuado um levantamento das características físicas e químicas do solo e uma avaliação quantitativa e qualitativa de fungos MA nativos, em área de cerrado natural e três áreas degradadas (cascalheiras I e II, e estrada rudimentar), do Parque Nacional de Brasília. Foram incluídas também parcelas de um experimento na cascalheira I, instalado em dezembro de 1993, como parte do Projeto de Recuperação de Áreas Degradadas-Convênio Ibama/Fub n 31/92 (Martins, 1996), para avaliar o estabelecimento da vegetação espontânea nas áreas degradadas do Parque. Esse experimento englobava os seguintes tratamentos: controle, calcário, turfa natural (humutrin), torta de mamona, humutrin + calcário e torta + calcário, todos aplicados na dose de $5 \mathrm{t} \mathrm{ha}^{-1} \mathrm{com}$ quatro repetições por tratamento.

Amostras de solo compostas foram coletadas aleatoriamente, no final dos períodos seco (setembro de 1994) e chuvoso (fevereiro de 1995), na profundidade de 0-5 cm. A amostragem foi efetuada na camada arável no cerrado natural e no subsolo das áreas degradadas, pois as camadas superiores haviam sido removidas.

Nas amostras foram determinados: a textura do solo, pelo método da dispersão total, a capacidade de campo pelo método da centrifugação utilizando-se amostras indeformadas, o pH em água $(1: 2,5)$ e os teores de $\mathrm{Al}, \mathrm{P}$, $\mathrm{K}$, Ca e Mg do solo, segundo Embrapa (1979). As determinações do $\mathrm{pH}$ do solo em $\mathrm{CaCl}_{2}$ e da acidez titulável $(\mathrm{H}+\mathrm{Al})$ foram efetuadas seguindo a metodologia de Raij et al. (1987).

As avaliações quantitativa e qualitativa de fungos MA nativos nas amostras foram efetuadas mediante a recuperação dos esporos dos fungos MA, pela técnica modificada de peneiramento úmido e decantação (Gerdemann \& Nicolson, 1963) e centrifugação (Coolen, 1979). A avaliação da colonização radicular foi feita pelos métodos de coloração (Phillips \& Hayman, 1970) e contagem (Giovannetti \& Mosse, 1980).

Os substratos coletados no final do período seco foram utilizados para a montagem de dois experimentos em casa de vegetação, conduzidos, de novembro de 1994 a fevereiro de 1995, na Embrapa-Centro de Pesquisa Agropecuária dos Cerrados (CPAC). Nos dois experimentos foi cultivada a gramínea nativa e pioneira de cerrado, Aristida setifolia Kunth, de ocorrência espontânea nas áreas em estudo (Martins, 1996). 
No primeiro experimento foram incluídos os substratos do cerrado natural, das cascalheiras I e II, e da estrada. Os substratos foram divididos e uma parte foi esterilizada a vapor. Os substratos natural e esterilizado foram colocados em vasos de $1 \mathrm{dm}^{3}$, que receberam duas plantas da gramínea nativa, pré-germinadas por 30 dias em areia esterilizada. Os substratos esterilizados receberam filtrados dos respectivos substratos naturais. Utilizou-se um delineamento inteiramente casualizado com três repetições.

No segundo experimento foram utilizados os substratos provenientes do experimento instalado no campo na cascalheira I, com a utilização de calcário e insumos orgânicos. Os substratos foram esterilizados a vapor, com posterior aplicação de seus respectivos filtrados e colocados em vasos de $1 \mathrm{dm}^{3}$. Foram plantadas duas plantas pré-germinadas da gramínea nativa, sem inoculação (NI) ou com inoculação (I) de uma mistura de fungos MA nativos de cerrado (Gigaspora margarita, Acaulospora scrobiculata e Glomus sp. CPAC2), oriundos da coleção da Embrapa-CPAC. Os tratamentos sem inoculação receberam filtrados do inoculante; utilizou-se um delineamento experimental inteiramente casualizado com três repetições.

Nos dois experimentos, as plantas foram cultivadas por um período de 90 dias, avaliando-se, então, as características químicas dos substratos, a altura e a produção de matéria seca, o número de esporos dos fungos MA no solo e a colonização radicular das plantas.

Os dados foram analisados estatisticamente pelos procedimentos do SAS (SAS Institute, 1990). As análises de variância foram executadas e as médias comparadas pelo teste de Duncan a $5 \%$ de probabilidade. Os dados de esporos foram transformados em $\mathrm{y}=(\mathrm{x}+0,5)^{0,5}$ e os de colonização radicular em $\mathrm{y}=\operatorname{arc} \operatorname{sen}(\mathrm{x} / 100)^{0,5}$.

\section{RESULTADOS E DISCUSSÃO}

As principais características físicas dos substratos coletados estão apresentadas na Tabela 1 . O teor de argila variou entre 395 a $500 \mathrm{~g} \mathrm{dm}^{-3}$, e a densidade aparente nas áreas degradadas foi superior à do cerrado natural, provavelmente porque o fluxo de veículos nessas áreas foi intenso. Esse aumento da densidade aparente pode causar um impedimento mecânico ao desenvolvimento do sistema radicular das plantas, bem como prejudicar a atividade microbiana pela deficiência de ar e água no solo (Baena \& Dutra, 1982).

Os dados da análise química (Tabela 2) mostram que as condições de acidez elevada, e baixa fertilidade, em todas as áreas, dificultariam o crescimento de plantas nesses substratos. $\mathrm{O}$ alto teor de matéria orgânica no solo de cerrado natural decorre, provavelmente, do grande acúmulo de biomassa por causa da colonização intensa da área por várias espécies de gramíneas nativas (Souza, 1991; Martins, 1996).

$\mathrm{Na}$ área degradada da cascalheira I, Leite et al. (1994) observaram também uma alta compactação do solo, com densidade de $1,6 \mathrm{~g} \mathrm{~cm}^{-3}$, com alta resistência à penetração na camada de $0-20 \mathrm{~cm}$ e baixa infiltração de água. Aliadas à baixa fertilidade do solo, principalmente quanto ao teor de $\mathrm{P}$ disponível para as plantas, essas características poderiam estar impedindo a revegetação do local. Esses autores sugerem que a combinação de práticas mecânicas para eliminar as restrições físicas e a adição de matéria orgânica seriam suficientes para iniciar o processo de colonização espontânea da área por plantas. Tais práticas poderiam também beneficiar a multiplicação dos fungos MA nativos, pois, segundo Veenendaal (1991), o escorrimento superficial da água da chuva assim como a erosão podem reduzir o número de propágulos desses fungos no solo e, conseqüentemente, limitar a sua propagação.

TABELA 1. Dados de análise granulométrica e de densidade aparente dos substratos nas áreas de cerrado natural (CN), cascalheira I (CI), cascalheira II (CII) e estrada (ES), do Parque Nacional de Brasília.

\begin{tabular}{|c|c|c|c|c|c|}
\hline Substrato & Argila & Silte & Areia fina & Areia grossa & Dens. aparente \\
\hline & |----------. & - & ) - & 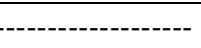 & $\left(\mathrm{g} \mathrm{cm}^{-3}\right)$ \\
\hline $\mathrm{CN}$ & 500 & 275 & 180 & 45 & 0,83 \\
\hline CI & 440 & 335 & 180 & 50 & 1,40 \\
\hline CII & 395 & 290 & 210 & 105 & 1,19 \\
\hline ES & 410 & 185 & 215 & 190 & 1,15 \\
\hline
\end{tabular}


Devido a essas características físicas e químicas, o crescimento de plantas poderia depender mais da associação micorrízica nos substratos degradados do que no solo de cerrado natural. Nessas condições, as gramíneas não colonizadas pelos fungos MA teriam maiores dificuldades para absorver o $\mathrm{P}$ e água do solo. Read et al. (1976) mostraram um aumento na absorção de $\mathrm{P}$ e água pelas plantas nativas colonizadas. Por outro lado, St. John (1982) sugere que, em ambientes com baixa disponibilidade de nutrientes, as plantas nativas são capazes de desenvolver estratégias para reduzir as exigências de nutrientes.

Os dados de análise dos substratos do experimento de campo da cascalheira I mostram que a aplica- ção de calcário e dos adubos orgânicos promoveu um aumento do $\mathrm{pH}$ em todos os tratamentos assim como do teor de $\mathrm{Ca}$ e $\mathrm{Mg}$ do solo (Tabela 3). O mesmo ocorreu com o teor de matéria orgânica e de $\mathrm{P}$, nos tratamentos que receberam humutrin e torta de mamona, que pode ter sido proveniente dos insumos utilizados, conforme mostram os dados da análise química na Tabela 4.

Os dados obtidos indicam a presença de fungos MA nas diversas áreas degradadas e de cerrado natural, ocorrendo um maior número de esporos no período chuvoso (Tabela 5). Segundo Maschio et al. (1992), os fungos MA têm adaptabilidade para colonizarem áreas que apresentem condições físicas, químicas e biológicas adversas e dependem

TABELA 2. Dados de análise química dos substratos nas áreas de cerrado natural (CN), cascalheira I (CI), cascalheira II (CII) e estrada (ES), do Parque Nacional de Brasília.

\begin{tabular}{|c|c|c|c|c|c|c|c|}
\hline Substrato & $\begin{array}{c}p H \\
\mathrm{H}_{2} \mathrm{O}(1: 2,5)\end{array}$ & $\begin{array}{c}\mathrm{pH} \\
\mathrm{CaCl}_{2}\end{array}$ & $\mathrm{Al}$ & $\mathrm{Ca}+\mathrm{Mg}$ & $\mathrm{P}$ & K & MO \\
\hline & & & \multicolumn{2}{|c|}{---- $\left(\mathrm{mmol}_{\mathrm{c}} \mathrm{kg}^{-1}\right)-$} & \multicolumn{2}{|c|}{--- $\left(\mathrm{mg} \mathrm{kg}^{-1}\right)$} & $\left(\mathrm{g} \mathrm{kg}^{-1}\right)$ \\
\hline $\mathrm{CN}$ & 5,3 & 4,2 & 3,9 & 18 & 1,1 & 69 & 45 \\
\hline CI & 5,2 & 4,7 & 0,6 & 4 & 0,6 & 23 & 10 \\
\hline CII & 5,3 & 4,3 & 3,4 & 9 & 0,8 & 69 & 16 \\
\hline ES & 5,1 & 4,5 & 2,1 & 4 & 0,8 & 23 & 17 \\
\hline
\end{tabular}

TABELA 3. Dados de análise química dos substratos da área experimental da cascalheira I, nas parcelas dos diversos tratamentos ${ }^{1}$.

\begin{tabular}{|c|c|c|c|c|c|c|}
\hline Tratamento & $\begin{array}{c}\mathrm{pH} \\
\mathrm{H}_{2} \mathrm{O}(1: 2,5)\end{array}$ & Al & $\mathrm{Ca}+\mathrm{Mg}$ & $\mathrm{P}$ & $\mathrm{K}$ & MO \\
\hline & & \multicolumn{2}{|c|}{-... $\left(\mathrm{mmol}_{\mathrm{c}} \mathrm{kg}^{-1}\right)$} & \multicolumn{2}{|c|}{ - $\left(\mathrm{mg} \mathrm{kg}^{-1}\right)$} & $\left(\mathrm{g} \mathrm{kg}^{-1}\right)$ \\
\hline Controle & $5,3 c$ & $0,2 \mathrm{a}$ & $7 \mathrm{c}$ & $0,5 \mathrm{e}$ & $5,0 \mathrm{e}$ & $11 \mathrm{c}$ \\
\hline Calcário & $7,2 \mathrm{a}$ & $0,1 \mathrm{a}$ & $34 \mathrm{a}$ & $0,8 \mathrm{~d}$ & $1,3 \mathrm{f}$ & $11 \mathrm{c}$ \\
\hline Humutrin & $6,9 \mathrm{~b}$ & $0,2 \mathrm{a}$ & $24 b$ & $1,7 \mathrm{c}$ & $18,6 \mathrm{c}$ & $16 \mathrm{a}$ \\
\hline Torta de mamona & $7,0 \mathrm{~b}$ & $0,1 \mathrm{a}$ & $28 \mathrm{~b}$ & $2,4 b$ & $40,6 \mathrm{a}$ & $16 \mathrm{a}$ \\
\hline Humutrin + calcário & $6,9 \mathrm{~b}$ & $0,2 \mathrm{a}$ & $40 \mathrm{a}$ & $1,7 \mathrm{c}$ & $8,7 \mathrm{~d}$ & $14 \mathrm{~b}$ \\
\hline Torta + calcário & $7,3 \mathrm{a}$ & $0,2 \mathrm{a}$ & $35 \mathrm{a}$ & $3,3 \mathrm{a}$ & $26,0 \mathrm{~b}$ & $12 \mathrm{c}$ \\
\hline
\end{tabular}

${ }^{1}$ Médias seguidas pela mesma letra na coluna não diferem entre si $(\mathrm{p}<0,05)$ pelo teste de Duncan.

TABELA 4. Dados de análise química dos insumos orgânicos aplicados na área experimental da cascalheira I.

\begin{tabular}{|c|c|c|c|c|c|c|c|c|c|c|}
\hline Insumo & $\mathrm{N}$ & $\mathrm{P}$ & $\mathrm{K}$ & $\mathrm{Ca}$ & $\mathrm{Mg}$ & $\mathrm{Fe}$ & $\mathrm{Cu}$ & $\mathrm{Zn}$ & $\mathrm{Mn}$ & $\mathrm{MO}$ \\
\hline & $---\cdot$ & - & $(\mathrm{g} \mathrm{k}$ & 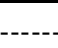 & & & $--(\mathrm{m}$ & $1)-$ & & $\left(\mathrm{g} \mathrm{kg}^{-1}\right)$ \\
\hline Humutrin & 40 & 5 & 6 & 40 & 21 & 660 & 18 & 71 & 210 & 750 \\
\hline Torta de mamona & 8 & 2 & 2 & 32 & 10 & 802 & 113 & 46 & 107 & 260 \\
\hline
\end{tabular}


TABELA 5. Número de esporos (E), colonização radicular (CR) e espécies predominantes de fungos MA nos substratos de cerrado natural (CN), cascalheira I (CI), cascalheira II (CII) e estrada (ES), nos períodos seco (SE) e chuvoso (CH) no campo ${ }^{1}$.

\begin{tabular}{|c|c|c|c|c|c|c|}
\hline \multirow[t]{2}{*}{ Substrato } & \multicolumn{2}{|c|}{$\mathrm{E}$} & \multicolumn{2}{|c|}{$\mathrm{CR}$} & \multicolumn{2}{|c|}{ Espécies de fungos $\mathrm{MA}^{2}$} \\
\hline & SE & $\mathrm{CH}$ & SE & $\mathrm{CH}$ & SE & $\mathrm{CH}$ \\
\hline & \multicolumn{2}{|c|}{--- $\left(\mathrm{n}^{\mathrm{o}} / 50 \mathrm{~g}\right)$--- } & & $(\%)$ & & \\
\hline $\mathrm{CN}$ & $181 \mathrm{a}$ & $260 \mathrm{a}$ & - & $62 \mathrm{a}$ & A. sp.; LOCT; C. sp. & A. sp.; E. sp.; L. sp.; G. sp.; C. sp. \\
\hline CI & $31 \mathrm{~b}$ & $232 \mathrm{a}$ & - & $41 \mathrm{a}$ & A. sp.; E .sp.; C. sp. & E. sp.; ECLB; C. sp. \\
\hline CII & $39 b$ & $305 a$ & - & $38 \mathrm{a}$ & A. sp.; C. sp. & A. sp.; ECLB; L. sp.; GMRG; C. sp. \\
\hline ES & $64 \mathrm{ab}$ & $227 \mathrm{a}$ & - & $43 a$ & A. sp.; G. sp.; C. sp. & ECLB; L. sp.; C. sp. \\
\hline
\end{tabular}

${ }^{1}$ Médias seguidas de mesma letra em cada parâmetro não diferem entre si $(\mathrm{p}<0,05)$ pelo teste de Duncan.

2 A. sp.: Acaulospora sp.; E. sp.: Entrophospora sp.; ECLB: Entrophospora colombiana; L. sp.: Glomus sp.; LOCT: Glomus occultum; G. sp.: Gigaspora sp.; GMRG: Gigaspora margarita; C. sp.: Scutellospora sp.

da umidade do solo para a sua multiplicação. Allen \& Allen (1980) citam, também, que a maioria das plantas que ocorrem espontaneamente em áreas mineradas apresentam simbiose com fungos MA e são beneficiadas no seu crescimento e sobrevivência. Dados experimentais obtidos por Kiernan et al. (1983) em uma área de mina de carvão abandonada e em processo de revegetação espontânea mostram a presença de fungos MA bem adaptados, apresentando multiplicação de propágulos no solo e formação de micorriza com as raízes das plantas.

O número de esporos no solo variou entre os períodos seco e chuvoso, também, em cada substrato, mas essa variação foi significativa somente nas áreas degradadas. No período seco, houve um número de esporos significativamente maior no solo do cerrado natural. Entretanto, essa diferença desapareceu no período chuvoso, quando as áreas degradadas apresentaram valores semelhantes aos do cerrado natural. No período chuvoso, o número de esporos no solo de cerrado natural aumentou de 1,5 vezes em relação ao período seco, ao passo que esse aumento foi de 7,7 vezes nos substratos das cascalheiras, e de 3,5 vezes no substrato da estrada. Esse tipo de variação sazonal foi constatado também por Miranda (1981) em área de cerrado cultivada com Brachiaria humidicola. Essas alterações durante as estações do ano decorrem principalmente das flutuações de precipitação pluvial (Allen \& Allen, 1980).
Nos substratos foram detectados, também, esporos de diferentes espécies de fungos MA. Algumas espécies, identificadas como Gigaspora margarita e Entrophospora colombiana, são de ocorrência comum em solos de cerrado, sendo usual a presença simultânea de várias espécies em uma única área ou rizosfera (Miranda \& Miranda, 1997). Dentre as espécies encontradas, Scutellospora sp. ocorreu nas quatro áreas e nos dois períodos de amostragem e a espécie Glomus occultum foi detectada apenas no cerrado natural e especificamente no período seco (Tabela 5). Em algumas áreas, a espécie Acaulospora sp. foi detectada apenas no período seco. Santos et. al. (1997) mostraram a presença de diferentes espécies de fungos MA em dunas de rejeito, e Kiernan et al. (1983) observaram também uma variação no número de espécies de fungos MA, com uma média de 3,2 espécies por amostragem, tendo encontrado até oito espécies nos melhores sítios.

No período chuvoso, a colonização radicular das plantas de cerrado natural de $62 \%$ não diferiu significativamente das outras áreas, que mantiveram um valor médio de 38\% a 43\% (Tabela 5). Entretanto, esses valores foram maiores que o observado por Veenendaal (1991) em plantas do mesmo gênero (Aristida congesta) em solos semi-áridos e degradados de Botswana, que apresentavam um valor médio de $28 \%$ de colonização radicular aos 80 dias após o seu estabelecimento. 
No levantamento realizado no experimento instalado em campo na cascalheira I, constatou-se que a população dos fungos MA foi baixa em todos os tratamentos, sendo mais elevada no tratamento com humutrin no período chuvoso (Tabela 6). Observou-se também que somente a espécie Acaulospora sp. estava presente em todos os tratamentos no período seco; no período chuvoso foi observada a ocorrência de até três espécies diferentes, e em alguns tratamentos não foi detectada a presença da Acaulospora sp. Outros dados obtidos por Martins (1996) mostram que esses tratamentos afetaram o estabelecimento espontâneo das plantas, produzindo um índice de cobertura de 56 indivídu$\mathrm{os} / \mathrm{m}^{2}$ nas parcelas com humutrin, 23 indivíduos $/ \mathrm{m}^{2}$ com calcário, 29 indivíduos $/ \mathrm{m}^{2}$ com torta+calcário e cerca de 11 indivíduos $/ \mathrm{m}^{2}$ nos demais tratamentos. Portanto, a variabilidade na população dos fungos MA nativos poderia estar relacionada também à variação na cobertura vegetal que afeta diretamente a multiplicação dos fungos (Sieverding,1991). Essas diferentes densidades de cobertura vegetal decorrentes dos tratamentos estariam somando seus efeitos aos dos próprios tratamentos na população dos fungos MA. O efeito benéfico da adubação orgânica aplicada em campo na população de fungos MA no solo foi também constatado por Baby \& Manibhushanrao (1996) em plantas de arroz infectadas com fungos MA, onde os vários adubos orgânicos utilizados favoreceram, em geral, a multiplicação dos esporos introduzidos no solo.
A aplicação do calcário no solo não alterou significativamente a população dos fungos MA nativos, mesmo na combinação com adubos orgânicos, embora mostre uma tendência de decréscimo no número de esporos, principalmente no período chuvoso. Reduções significativas na densidade de esporos de fungos MA nativos em função de níveis altos de calagem têm sido observadas em outros trabalhos. Miranda et al. (1997) mostram que a população de fungos MA nativos aumentou até a dose de $4 \mathrm{t} / \mathrm{ha}$ de calcário aplicado em um solo glei pouco húmico, decrescendo nas doses mais elevadas. Sieverding (1991) observou esse decréscimo sugerindo a ocorrência de diferentes graus de sensibilidade à calagem entre espécies de fungos MA e que a calagem pode interferir também no número de espécies presentes no solo.

No primeiro experimento, em casa de vegetação, a presença dos fungos MA nativos no cerrado natural e nas áreas degradadas, promoveu um aumento significativo na altura e na produção de matéria seca da gramínea nativa Aristida setifolia (Tabela 7). As condições de melhor fertilidade e maior teor de matéria orgânica da camada arável do cerrado natural poderiam ter contribuído para o maior crescimento das plantas observado nesse substrato. Entretanto, na ausência dos fungos MA, a altura e a produção de matéria seca da gramínea foram muito pequenas e semelhantes em todos os substratos. De acordo com os dados da Tabela 2, haveria uma limitação ao desenvolvimento de plantas em decorrência do baixo nível de P disponí-

TABELA 6. Número de esporos (E) e espécies predominantes de fungos MA nos substratos da cascalheira I, com diversos tratamentos, nos períodos seco $(\mathrm{SE})$ e chuvoso $(\mathrm{CH})$ no campo ${ }^{1}$.

\begin{tabular}{|c|c|c|c|c|}
\hline \multirow[t]{2}{*}{ Tratamento } & \multicolumn{2}{|c|}{$\mathrm{E}$} & \multicolumn{2}{|c|}{ Espécies de fungos $\mathrm{MA}^{2}$} \\
\hline & SE & $\mathrm{CH}$ & $\mathrm{SE}$ & $\mathrm{CH}$ \\
\hline \multicolumn{5}{|c|}{ - } \\
\hline Controle & $3 b$ & $7 \mathrm{ab}$ & A. sp. & A. sp.; L. sp.; G. sp. \\
\hline Calcário & $1 b$ & $7 \mathrm{ab}$ & A. sp. & LCLR \\
\hline Humutrin & $11 \mathrm{ab}$ & $171 \mathrm{a}$ & A. sp. & E. sp.; L. sp.; LCLR \\
\hline Torta de mamona & $0 b^{3}$ & $44 \mathrm{ab}$ & A. $\mathrm{sp}$. & A.sp.; L. sp.; GMRG \\
\hline Humutrin + Calcário & $0 b^{3}$ & $51 \mathrm{a}$ & A. $\mathrm{sp}$. & A. sp.; L. sp. \\
\hline Torta + Calcário & $1 b$ & $10 \mathrm{ab}$ & A. sp. & A. sp.; L. sp.; G. sp. \\
\hline
\end{tabular}


TABELA 7. Altura de plantas (Alt.), produção de matéria seca (MS), número de esporos (E) e colonização radicular (CR) da gramínea Aristida setifolia cultivada em solo esterilizado (Est) e natural (Nat) de cerrado e de áreas degradadas, em casa de vegetação ${ }^{1}$.

\begin{tabular}{cccccc}
\hline Substrato $^{2}$ & Tratamento & Alt. & MS & E & CR \\
\hline \multirow{2}{*}{ CN } & & $(\mathrm{cm})$ & $(\mathrm{mg} / \mathrm{vaso})$ & $\left(\mathrm{n}^{\circ} / 50 \mathrm{~g}\right)$ & $(\%)$ \\
& Est & $0,4 \mathrm{~d}$ & $5,7 \mathrm{e}$ & $0 \mathrm{~b}$ & $0,0 \mathrm{~d}$ \\
$\mathrm{CI}$ & Nat & $36,7 \mathrm{a}$ & $590,7 \mathrm{a}$ & $56 \mathrm{a}$ & $44,6 \mathrm{a}$ \\
& Est & $1,4 \mathrm{~d}$ & $5,9 \mathrm{e}$ & $0 \mathrm{~b}$ & $0,0 \mathrm{~d}$ \\
& Nat & $6,7 \mathrm{c}$ & $31,3 \mathrm{~d}$ & $64 \mathrm{a}$ & $33,6 \mathrm{ab}$ \\
& Est & $1,3 \mathrm{~d}$ & $11,6 \mathrm{c}$ & $0 \mathrm{~b}$ & $0,0 \mathrm{~d}$ \\
& Nat & $9,7 \mathrm{c}$ & $49,0 \mathrm{c}$ & $69 \mathrm{a}$ & $19,0 \mathrm{c}$ \\
& Est & $1,3 \mathrm{~d}$ & $6,7 \mathrm{e}$ & $0 \mathrm{~b}$ & $0,0 \mathrm{~d}$ \\
& Nat & $13,6 \mathrm{~b}$ & $87,7 \mathrm{~b}$ & $58 \mathrm{a}$ & $56,0 \mathrm{a}$ \\
\hline
\end{tabular}

${ }^{1}$ Médias seguidas pela mesma letra na coluna não diferem entre si $(\mathrm{p}<0,05)$ pelo teste de Duncan.

${ }^{2} \mathrm{CN}$ : cerrado natural; CI: cascalheira I; CII: cascalheira II; E: estrada.

vel, em todos os substratos utilizados, o que reforça a contribuição dos fungos MA no crescimento da gramínea nativa. Miller (1979), ao estudar a ocorrência de fungos MA em áreas naturais e mineradas do deserto de Red, nos EUA, constatou que nos locais com baixo nível de $\mathrm{P}$ ocorreu uma seleção entre plantas, havendo maior desenvolvimento para aquelas que formavam micorrizas. Observou-se, também, que o número de esporos de fungos MA nos tratamentos sem esterilização foi semelhante em todos os substratos, porém a colonização radicular mostrou alguma variabilidade e foi significativamente inferior nas plantas da cascalheira II.

No segundo experimento, em casa de vegetação, com o solo degradado da cascalheira I, a aplicação prévia, no campo, de calcário e de adubos orgânicos contribuíu pouco para o crescimento da gramínea (Tabela 8), e não houve diferença significativa na altura e produção de matéria seca das plantas entre os tratamentos não infectados. Entretanto, a inoculação de fungos MA nativos promoveu um aumento significativo nesses parâmetros, assim como uma diferenciação de efeitos entre os insumos utilizados. O maior efeito foi observado nos tratamentos com os insumos orgânicos, humutrin e torta de mamona, provavelmente devido ao maior teor de $\mathrm{P}$ contido nesses insumos, conforme mostrado nas Tabelas 3 e 4 . A adição de calcário, isoladamente ou combinado com os insumos orgânicos, causou uma redução nesses efeitos, como já observado anteriormente na população dos fungos MA em campo. Segundo Howeler et al. (1987), Jasper et al. (1988) e Miranda \& Miranda (1997), vários fatores podem afetar a eficiência dos fungos $\mathrm{MA}$, destacando-se o $\mathrm{pH}$ e os níveis de $\mathrm{P}$ e $\mathrm{K}$ do substrato.

No tratamento controle não inoculado (Tabela 8), os dados foram semelhantes aos obtidos com o mesmo substrato da cascalheira I esterilizado no primeiro experimento em casa de vegetação (Tabela 7). Porém, observa-se um efeito maior da micorriza, na altura e produção de matéria seca das plantas, com a inoculação da mistura de fungos MA do que no mesmo substrato com fungos nativos. Este efeito deve-se, provavelmente, à presença da espécie Glomus sp. CPAC2, presente no inoculante e ausente no substrato, ou à posição localizada do inoculante, no segundo experimento, pois em ambos os experimentos a micorriza foi estabelecida por espécies de fungos MA nativas de cerrado. Quanto ao número de esporos e à colonização radicular das plantas nos tratamentos infectados, embora as diferenças não tenham sido significativas em relação ao número de esporos no solo, ambos os parâmetros foram maiores no tratamento com torta, em que a inoculação promoveu a maior altura das plantas e produção de matéria seca.

Os dados experimentais obtidos ressaltam a contribuição da micorriza no estabelecimento de plantas em solos degradados e corroboram as observações de outros autores (Gardner \& Malajczuk, 1988; Jasper et al., 1988; Sylvia, 1990; Franco et al., 1992; Raman et al., 1993; Carneiro et al., 1997), que demonstraram a importância dos fungos MA no processo de recuperação de áreas degradadas. 
TABELA 8. Altura de plantas (Alt.), produção de matéria seca (MS), número de esporos (E) e colonização radicular (CR) da gramínea Aristida setifolia, cultivada nos substratos esterilizados da cascalheira I, com diversos tratamentos prévios no campo, sem inoculação (NI) e com inoculação (I) de uma mistura de fungos MA nativos de cerrado, em casa de vegetação ${ }^{1}$.

\begin{tabular}{lccccc}
\hline Tratamento & Inoc. $^{2}$ & Alt. & MS & E & CR \\
\hline \multirow{4}{*}{ Controle } & & $(\mathrm{cm})$ & $(\mathrm{mg} / \mathrm{vaso})$ & $\left(\mathrm{n}^{\circ} / 50 \mathrm{~g}\right)$ & $(\%)$ \\
& $\mathrm{NI}$ & $0,4 \mathrm{~cd}$ & $5,2 \mathrm{bc}$ & $0 \mathrm{~d}$ & $0,0 \mathrm{~d}$ \\
Calcário & $\mathrm{I}$ & $11,8 \mathrm{~b}$ & $113,9 \mathrm{~b}$ & $88 \mathrm{~b}$ & $17,0 \mathrm{c}$ \\
& $\mathrm{NI}$ & $0,4 \mathrm{~cd}$ & $8,7 \mathrm{bc}$ & $0 \mathrm{~d}$ & $0,0 \mathrm{~d}$ \\
Humutrin & $\mathrm{I}$ & $6,7 \mathrm{bc}$ & $56,9 \mathrm{bc}$ & $42 \mathrm{bc}$ & $14,7 \mathrm{c}$ \\
& $\mathrm{NI}$ & $0,4 \mathrm{~cd}$ & $7,2 \mathrm{bc}$ & $0 \mathrm{~d}$ & $0,0 \mathrm{~d}$ \\
Torta & $\mathrm{I}$ & $20,9 \mathrm{~b}$ & $434,0 \mathrm{~b}$ & $116 \mathrm{ab}$ & $28,0 \mathrm{~b}$ \\
& $\mathrm{NI}$ & $0,6 \mathrm{~cd}$ & $8,2 \mathrm{bc}$ & $0 \mathrm{~d}$ & $0,0 \mathrm{~d}$ \\
Humut. + calcário & $\mathrm{I}$ & $35,1 \mathrm{a}$ & $1109,9 \mathrm{a}$ & $335 \mathrm{a}$ & $43,0 \mathrm{a}$ \\
& $\mathrm{NI}$ & $0,5 \mathrm{~cd}$ & $7,7 \mathrm{bc}$ & $0 \mathrm{~d}$ & $0,0 \mathrm{~d}$ \\
Torta + calcário & $\mathrm{I}$ & $15,8 \mathrm{~b}$ & $79,5 \mathrm{bc}$ & $305 \mathrm{a}$ & $31,7 \mathrm{~b}$ \\
& $\mathrm{NI}$ & $1,4 \mathrm{c}$ & $7,2 \mathrm{bc}$ & $0 \mathrm{~d}$ & $0,0 \mathrm{~d}$ \\
& $\mathrm{I}$ & $20,6 \mathrm{~b}$ & $378,9 \mathrm{~b}$ & $154 \mathrm{ab}$ & $30,7 \mathrm{~b}$ \\
\hline
\end{tabular}

${ }^{1}$ Médias seguidas pela mesma letra na coluna não diferem entre si $(\mathrm{p}<0,05)$ pelo teste de Duncan.

2 Mistura dos fungos MA: Glomus sp. CPAC2, Gigaspora margarita e Acaulospora scrobiculata.

\section{CONCLUSÕES}

1. Os fungos micorrízicos arbusculares estão presentes nas áreas degradadas e sua população apresenta variação sazonal.

2. Os fungos MA nativos contribuem significativamente para o estabelecimento da gramínea nativa e pioneira Aristida setifolia nos solos de cerrado natural e de áreas degradadas.

3. A adição de calcário e dos insumos orgânicos humutrin e torta de mamona não influencia no crescimento da gramínea cultivada em solo degradado de cascalheira, na ausência de fungos MA.

4. A inoculação de fungos MA nativos de cerrado em solo degradado de cascalheira promove o estabelecimento da gramínea nesse solo e maximiza os efeitos do calcário e dos insumos orgânicos utilizados.

\section{REFERÊNCIAS}

ALLEN, E.B.; ALLEN, M.F. Competition between plants of different successional stages: mycorrhizae as regulators. Canadian Journal of Botany, Ottawa, v.62, p.2625-2629, 1984.
ALLEN, E.B.; ALLEN, M.F. Natural re-establishment of vesicular-arbuscular mycorrhiza following strip mine reclamation in Wyoming. Journal of Applied Ecology, Oxford, v.17, p.139-147, 1980.

BABY, U.I.; MANIBHUSHANRAO, K. Influence of organic amendments on arbuscular mycorrhizal fungi in relation to rice sheath blight disease. Mycorrhiza, Secaucus, v.6, p.201-206, 1996.

BAENA, A.R.C.; DUTRA, S. Propriedades físicas dos principais solos da Amazônia brasileira em condições naturais. Belém: Embrapa-CPATU, 1982. 28p. (Embrapa-CPATU. Boletim de Pesquisa, 33).

CHIOSSI, N.J. Ocupação do solo e impacto ambiental. Revista Brasileira de Tecnologia, Brasília, v.13, n.5, p.44-51, 1982.

CARNEIRO, M.A.C.; SIQUEIRA, J.O.; CURI, N.; MOREIRA, F.M.S. Fungos micorrízicos e superfosfato no acúmulo de nitrogênio e fósforo e no crescimento de plantas herbáceas em solo degradado. In: SIMPÓSIO BRASILEIRO DE RECUPERAÇÃO DE ÁREAS DEGRADADAS, 3., 1997, Ouro Preto. Trabalhos voluntários. Viçosa: SOBRADE/UFV/DPS/DEF, 1997. p.231-239.

COOLEN, W.R. Methods for the extraction of Meloidogyne spp. and other nematodes from roots and soil. In: LAMBERTI, F.; TAYLOR, C.E. (Eds.). 
Root-knot nematodes (Meloidogyne species): systematics, ecology and control. London: Academic Press, 1979. p.317-329.

EMBRAPA. Serviço Nacional de Levantamento e Conservação de Solos.( Rio de Janeiro, RJ). Manual de métodos de análises de solo. Rio de Janeiro, 1979. não paginado.

FRANCO, A.A.; CAMPELLO, E.F.; SILVA, E.M.R.; FARIA, S.M. Revegetação de solos degradados. Itaguai: Embrapa-CNPBS, 1992. 11p. (EmbrapaCNPBS. Comunicado técnico, 9).

GARDNER, J.H.; MALAJCZUK, N. Recolonisation of rehabilitated bauxite mine sites in Western Australia by mycorrhizal fungi. Forest, Ecology and Management, Amsterdam, v.24, p.27-42, 1988.

GERDEMANN, J.W.; NICOLSON, T.H. Spores of mycorrhizal endogone species extracted from soil by wet sieving and decanting. Transactions of the British Mycological Society, London, v.46, p.235-244, 1963.

GIOVANNETTI, M.; MOSSE, B. An evaluation of techniques to measuring vesicular arbuscular mycorrhizal infection in roots. New Phytologist, Oxford, v.84, p.489-500, 1980.

HOWELER, R.H.; SIEVERDING, E.; SAIF, S. Practical aspects of mycorrhizal technology in some tropical crops and pastures. Plant and Soil, Dordrecht, v.100, p.249-283, 1987

INSTITUTO BRASILEIRO DE MINERAÇÃO. Comissão Técnica de Meio Ambiente. Solo e biota. In: INSTITUTO BRASILEIRO DE MINERAÇÃO. Comissão Técnica de Meio Ambiente. Mineração e Meio Ambiente. Brasília, 1992. p.43-51.

JASPER, D.A.; ROBSON, A.D.; ABBOTT, L.K. Revegetation in an Iron-ore mine - Nutrient requirements for plant growth and the potential role of vesicular-arbuscular (VA) mycorrhizal fungi. Australian Journal of Soil Research, Victoria, v.26, p.497-507, 1988

KIERNAN, J.M.; HENDRIX, J.W.; MARONEK, D.M. Endomycorrhizal fungi occurring on orphan strip mines in Kentucky. Canadian Journal of Botany, Ottawa, v.61, p.1798-1803, 1983.

LEITE, L.L.; MARTINS C.R.; HARIDASAN, M Efeitos da descompactação e da adubação do solo na revegetação espontânea de uma cascalheira no
Parque Nacional de Brasília. In: SIMPÓSIO SUL-AMERICANO, 1.; SIMPÓSIO NACIONAL SOBRE RECUPERAÇÃO DE ÁREAS DEGRADADAS, 2., 1994, Foz do Iguaçu. Anais... Curitiba: UFPR/Fundação de Pesquisas Florestais do Paraná, 1994. p.527-534.

LEITE, L.L.; MARTINS C.R.; HARIDASAN, M. Propriedades físico-hídricas do solo de uma cascalheira e de áreas adjacentes com vegetação nativa de campo sujo e cerrado no Parque Nacional de Brasília. In: SIMPÓSIO NACIONAL SOBRE RECUPERAÇÃO DE ÁREAS DEGRADADAS, 1. 1992, Curitiba. Anais... Curitiba: UFPR/Fundação de Pesquisas Florestais do Paraná, 1992. p.392-399.

MARTINS, C.R. Revegetação com gramíneas de uma área degradada no Parque Nacional de Brasília, DF, Brasil. Brasília: UnB, 1996. 70p. Tese de Mestrado.

MASCHIO, L.; GAIAD, S.; MONTOYA, L.; CURCIO, G.R.; RACHWALL, M.F.G.; CAMARGO, C.M.S.; BATTI, A.M.B. Microrganismos e auto-sustentação de ecossistemas em solos alterados. In: SIMPÓSIO NACIONAL SOBRE RECUPERAÇÃO DE ÁREAS DEGRADADAS, 1992, Curitiba. Anais.. Curitiba: UFPR/Fundação de Pesquisas Florestais do Paraná, 1992. p.440-445.

MILLER, R.M. Some occurrences of vesicular-arbuscular mycorrhiza in natural and disturbed ecosystems of the Red Desert. Canadian Journal of Botany, Ottawa, v.57, p.619-623, 1979.

MIRANDA, J.C.C. Ocorrência de fungos endomicorrízicos nativos em um solo de Cerrado do Distrito Federal e sua influência na absorção de fósforo por Brachiaria decumbens Stapf. Revista Brasileira de Ciência do Solo, Campinas, v.5, n.2, p.102-105, 1981.

MIRANDA, J.C.C.; MIRANDA, L.N. Micorriza Arbuscular. In: VARGAS, M.A.; HUNGRIA, M., (Eds.). Biologia dos solos dos Cerrados. Brasília: Embrapa-CPAC, 1997. p.69-123.

MIRANDA, L.N; MIRANDA, J.C.C.; VIVALDI, L. Efeito residual do calcário e manejo da calagem em solo gley-pouco-húmico. Relatório Técnico Anual do Centro de Pesquisa Agropecuária dos Cerrados 1991/1995. Planaltina, 1997. p.82-83.

NEPSTAD, D.C.; UHL, C.; SERRÃO, E.A.S Recuperation of a degraded Amazonian landscape: forest recovery and agricultural restoration. Ambio: 
Journal of the Human Environment Research and Management, Stokholm, v.20, n.6, p.248-255, 1991.

PHILLIPS, J.M.; HAYMAN, D.S. Improved procedures for clearing and staining parasitic and vesiculararbuscular fungi for rapid assessment of infection. Transactions of the British Mycological Society, v.55, p.158-161, 1970.

RAIJ, B.van; QUAGGIO, J.A.; CANTARELLA, H.; FERREIRA, M.E.; LOPES, A.S.; BATAGLIA, O.C. Análise química do solo para fins de fertilidade. Campinas: Fund. Cargill, 1987. 170p.

RAMAN, N.; NAGARAJAN, N.; GOPINATHAN, S.; SAMBANDAN, K. Mycorrhizal status of plants species colonizing a magnesite mine spoil in India. Biology and Fertility of Soils, Heidelberg, v.16, p.76-78, 1993.

READ, D.J.; KOUCHEKI, H.K.; HODGSON, J. Vesicular-arbuscular mycorrhiza in natural vegetation systems. New Phytologist, Oxford, v.77, p.641-653, 1976.

RUIVO, M.L.P. Recuperação de áreas de mineração: uma experiência bem sucedida na Amazônia. In: FERREIRA, E.J.G.; SANTOS, G.M.; LEÃO, E.I.M.; OLIVEIRA, I.A. (Eds.). Bases científicas para estratégias de preservação e desenvolvimento da Amazônia. Manaus: INPA, 1993. p.383-404.

SANTOS, M.; ROSADO, S.C.S.; SIQUEIRA, J.O.; ORLANDINI, L. Fungos micorrízicos arbusculares em dunas de rejeito, sob processo de recuperação, no litoral norte da Paraíba. In: SIMPÓSIO BRASILEIRO DE RECUPERAÇÃO DE ÁREAS
DEGRADADAS, 3., 1997, Ouro Preto. Trabalhos voluntários. Viçosa: SOBRADE/ UFV/DPS/DEF, 1997. p.227-230.

SAS INSTITUTE. SAS/STAT user's guide. Cary, NC, 1990. v.1, 890p.

SIEVERDING, E. Vesicular-arbuscular mycorrhiza management in tropical agrosystems. Eschborn: GTZ, 1991. 371p.

SOUZA, F.H.D. Maturação e colheita de sementes de plantas forrageiras. Revista Brasileira de Sementes, Brasília, v.3, n.1, p.143-157, 1991.

ST. JOHN, T.V. The role of mycorrhizae in plant ecology. Canadian Journal of Botany, Ottawa, v.61, p.1005-1014, 1982.

SYLVIA, D.M. Inoculation of native woody plants with vesicular-arbuscular mycorrhizal fungi for phosphate mine land reclamation. Agriculture, Ecosystems and Environment, Amsterdam, v.31, p.253-261, 1990.

VEENENDAAL, E.M. Adaptive strategies of grasses in a semi-arid savannah in Botswana. Amsterdam: Vrije Universiteit van Amsterdam, 1991. 170p. Ph.D. Thesis.

VILELA, L.; BARCELLOS, A.O.; SANZONOWICZ, C.; SPAIN, J.M. Recuperação de pastagem de Brachiaria ruziziensis através do uso de grade aradora, nitrogênio e introdução de leguminosas. Relatório Técnico Anual do Centro de Pesquisa Agropecuária dos Cerrados 1985/1987. Planaltina, 1991. p.239-241. 